DOI: $10.26851 /$ RUCP.27.1

\title{
CONSTRUYENDO UN EQUILIBRIO DEMOCRÁTICO: LA REFORMA CONSTITUCIONAL DE URUGUAY EN $1917^{*}$
}

\author{
Building a Democratic Equilibrium. The Constitutional Reform of Uruguay in 1917
}

\section{Daniel Buquet ${ }^{1}$ y Juan Andrés Moraes ${ }^{2}$}

\begin{abstract}
Resumen: ¿Qué explica el nacimiento de una democracia moderna? ¿Por qué un pacto democrático sobre la base de reglas y procedimientos puede tornarse estable a través del tiempo? Aunque estas son preguntas fundamentales para el estudio de las democracias, Uruguay no cuenta aún con una explicación guiada teóricamente que dé cuenta del origen de la democracia en el país. Este trabajo explica cómo y por qué se formó la democracia en Uruguay, a partir de una modelación de las interacciones estratégicas entre los principales actores políticos en Uruguay de principios del siglo XX. Utilizando teoría de juegos, el trabajo analiza proceso de adopción de los principales aspectos del sistema electoral uruguayo en la Convención Nacional Constituyente entre 1916 y 1917 y muestra cómo esas normas generaron un equilibrio competitivo. Asimismo, mostramos que lo que caracteriza a la democracia uruguaya y le confiere altos niveles de estabilidad es la capacidad de sus actores políticos para pactar reglas que generan un amplio consenso. El trabajo se nutre de algunos datos descriptivos y una detallada narrativa histórica.
\end{abstract}

Palabras Clave: democracia, instituciones, sistema electoral, partidos políticos, Teoría de Juegos.

Summary: What explains the birth of a modern democracy? Why a democratic agreement based on rules and procedures can become stable over time? Although these are fundamental questions for the study of democracies, Uruguay still lacks a theoretically guided explanation that accounts for the origin of democracy. This paper explains how and why democracy was established in Uruguay, modeling the strategic interactions between the main political actors at the beginning of the 20th century. Using game theory, this work analyzes the process towards the adoption of the main features of the electoral system approved by the National Constituent Convention in 1917, and how these rules were able to produce a competitive equilibrium. Likewise, we show that what features Uruguayan democracy and its high levels of political stability is the ability of political elites to agree on rules that create a broad consensus. The work is empirically grounded in basic descriptive data and a detailed historical narrative.

Key words: democracy; institutions; electoral system; political parties; Game Theory.

\footnotetext{
* Artículo recibido el 30/11/2017 y aceptado el 21/03/2018.

1 Instituto de Ciencia Política, Facultad de Ciencias Sociales, Universidad de la República. Correo electrónico: $\underline{\text { dbuquet@gmail.com }}$

2 Instituto de Ciencia Política, Facultad de Ciencias Sociales, Universidad de la República. Correo electrónico: Juan.A.Moraes.1@nd.edu
} 


\section{Introducción}

Nos encontramos en un periodo en el que la democracia uruguaya puede legítimamente celebrar su primer centenario. No se trata de un acontecimiento menor ya que estamos hablando de la democracia más longeva de América Latina. También es cierto que el país no vivió un siglo continuado de democracia -casi ningún país en el mundo lo ha logrado- debido a las dos interrupciones que sufrió el régimen durante el siglo XX $\mathrm{XX}^{3}$. Pero los ańos que Uruguay ha vivido bajo un régimen democrático, que sumados superan los 75, también constituyen el mayor guarismo en Latinoamérica y supera a buena parte de los países de Europa Occidental.

El reconocimiento de semejante logro no busca, sin embargo, el regocijo autocomplaciente que tanto parece entusiasmar a muchos uruguayos. Lo relevante es saber que la democracia actual tiene aquel origen. Porque, a pesar de las interrupciones mencionadas, el sistema político uruguayo estableció, en aquella coyuntura, algunos de sus rasgos esenciales, cuya continuidad está en la base de su característica estabilidad política. Para entender cómo funciona este sistema tenemos que comprender cómo fue construido. En particular, la segunda década del siglo XX fue el periodo en el que se constituyó el sistema de partidos moderno en Uruguay, a través del reconocimiento mutuo de los actores y el establecimiento de reglas para la competencia política y el ejercicio del gobierno.

Las teorías sobre el origen de la democracia han hecho particular hincapié en dos tipos de explicaciones. Por un lado, las teorías económicas se centran en la estructura de clases y los conflictos distributivos, como base de las estrategias que los actores económicos proyectan en la distribución del poder político (Moore, 1966; Acemoglu y Robinson, 2003). Como regla, estos conflictos de clase suelen estar latentes en todos los regímenes políticos, en particular en las instancias de transición a la democracia desde regímenes autocráticos u oligarquías competitivas (Dahl, 1971) como la observada en Uruguay hacia 1917. En un plano similar, otras explicaciones estructuralistas se han concentrado en el efecto causado por el desarrollo socioeconómico y la modernización (Lipset, 1959) sobre la probabilidad de la democracia. Nuestro trabajo no rechaza esta clase de fenómenos ni la existencia de conflictos de clase más o menos manifiestos, sino que busca aislar las motivaciones e intereses políticos de los agentes en el proceso de negociación de reglas de juego democráticas. Por otro lado, teorías de corte culturalista han hecho hincapié en la estructura de preferencias de las elites y la forma en que estas se orientan a reconocer en la democracia a un conjunto de reglas para la resolución de conflictos. Desde esta perspectiva, la existencia de valores asociados al ejercicio de derechos políticos y civiles hace más probable la emergencia de regímenes democráticos (Almond y Verba, 1963). Nuestro trabajo tampoco descarta la influencia de factores asentados en la cultura política

3 La primera ocurrió en 1933 y fue un golpe de Estado civil que buscó una rápida re-institucionalización a través de reformas constitucionales y legales. Sin embargo, la falta de legitimidad de los dos gobiernos elegidos (1934 y 1938) en virtud de esas normas implicó que la democracia efectiva sólo se recuperara en 1942, con una nueva reforma constitucional. La segunda ruptura, entre 1973 y 1984, condujo a una dictadura militar similar a otras que asolaron el continente en ese momento. 
democrática de las elites, sino que asume que no es suficiente la existencia de estas preferencias para concretar un proyecto democrático.

En nuestro trabajo, la democracia es el fruto de la construcción de un equilibrio que las elites logran mediante la negociación de un conjunto de reglas que se fundamenta exclusivamente en su interés de participar en el gobierno. El enfoque que utilizamos se vincula más directamente con el utilizado para explicar las reformas electorales desde la perspectiva de los intereses de los reformadores (Benoit, 2004; Colomer, 2005) y, de forma más específica, con estudios sobre la adopción de la representación proporcional (Rokkan, 1970; Boix, 1999). Con este último enfoque, Wills-Otero (2009) propone que para América Latina se adoptó la representación proporcional cuando los partidos en el poder enfrentaron un desafío que los podía conducir a la derrota, como forma de retener una parte de poder. Sin embargo, recientemente, Negretto (2017) cuestiona la validez general del argumento e identifica tres caminos diferentes que conducen a la adopción de la representación proporcional en la región. En su estudio, la explicación de la adopción de la representación proporcional en Uruguay (al igual que en Colombia, Costa Rica y Panamá) ocurre como consecuencia de la aparición de una poderosa fracción disidente dentro del partido gobernante que busca competir más eficazmente. Aunque este trabajo buscar explicar la democratización en Uruguay y no simplemente la adopción de la representación proporcional, como se verá más adelante, tanto la incorporación de la representación proporcional como el papel jugado por la fracción disidente del Partido Colorado son aspectos centrales del argumento.

Este trabajo desarrolla una narrativa analítica (Bates et al., 1998) para explicar cómo se formó la democracia en Uruguay y, en particular, cómo se constituyó su núcleo central; un conjunto de reglas y procedimientos. Para ello, el trabajo parte de la identificación de lo que llamamos equilibrio inestable que caracterizó al siglo XIX, a partir de su primer texto constitucional de 1830. Específicamente, para explicar la reforma constitucional de 1917, nuestro trabajo repasa las características de la lucha por el poder entre los partidos durante la vigencia de la constitución de 1830. Luego analizamos el proceso de adopción de los principales aspectos del sistema electoral uruguayo a través de la Convención Nacional Constituyente entre 1916 y 1917. Esta narrativa constituye el insumo indispensable para la elaboración de una explicación basada en la teoría de juegos, capaz de captar el pasaje a un nuevo equilibrio marcado por la confección de reglas de juego consensuadas entre los partidos. Estas reglas establecidas en la reforma constitucional de 1917 pautan tanto el inicio como la continuidad de la democracia en Uruguay.

\section{Los antecedentes políticos}

La Constitución uruguaya de 1830 no preveía la existencia de partidos políticos y, tanto allí como en la ley electoral de abril del mismo año, se establecía un régimen electoral que no se adecuaba a una competencia pacífica por el poder. Sintéticamente, el sistema electoral que rigió durante casi todo el siglo XIX en Uruguay establecía 
un sistema mayoritario extremo para la elección de la Cámara de Representantes y un sistema mayoritario indirecto para la elección de la Cámara de Senadores. Los senadores eran electos a razón de uno por departamento a través de un colegio elector departamental y a cada departamento le correspondía un diputado "...por cada tres mil almas, o por una fracción que no baje de dos mil". El voto era emitido de viva voz, nombrando a los candidatos y el electorado era muy restringido dadas las condiciones que imponía la Constitución para el ejercicio del sufragio ${ }^{4}$. Finalmente, el Presidente de la República era electo indirectamente por la Asamblea General conformada por la reunión de ambas Cámaras.

Los diputados eran electos en circunscripciones plurinominales por el sistema mayoritario, lo que implicaba que la lista con más votos se quedara con la totalidad de los cargos en disputa. Los senadores eran electos en circunscripciones uninominales, pero a través de un colegio elector que también era electo por el mismo sistema mayoritario extremo. Este sistema no facilitaba -de hecho, normalmente impedía- la representación de las minorías, cuestión que, a su vez, tornaba las elecciones en acontecimientos conflictivos, permanentemente distorsionadas por la abstención, la injerencia del poder político, la violencia, la manipulación y el fraude. Más aún cuando la designación del Presidente de la República dependía de la integración del Poder Legislativo.

Los partidos tradicionales uruguayos se originaron en un conflicto entre los dos primeros presidentes constitucionales del país en 1836. Los bandos que se alinearon detrás de uno y otro caudillo evolucionaron con el tiempo en partidos políticos que convirtieron los colores de sus distintivos en sus denominaciones oficiales. Los conflictos entre el Partido Colorado y el Partido Blanco -posteriormente denominado Nacional- no tenían un carácter ideológico y su lucha por el poder político se desarrolló, hasta principios del siglo XX, en términos fundamentalmente bélicos.

La primera forma de coexistencia pacífica, relativamente institucionalizada, entre los partidos surgió en 1872, a partir del pacto que puso fin a la revolución conducida por el caudillo blanco Timoteo Aparicio. Dice Pivel Devoto (1994, p. 228), refiriéndose a aquél acuerdo, que se "...habia consagrado, en efecto, el principio de la coexistencia de los partidos mediante una fórmula de coparticipación política, en virtud de la cual al Partido Blanco le sería confiada la administración de cuatro Departamentos". Este tipo de acuerdos - primero informales, luego institucionalizados- constituyeron, a partir de entonces, una impronta característica del relacionamiento entre los partidos tradicionales. La coparticipación fue, en esa primera instancia, un sucedáneo de un mecanismo de representación de minorías. Cuando el Partido Blanco ejercía una jefatura departamental, no sólo tenía el control administrativo sobre una parte del territorio, sino que tenía -en consecuencia- amarrada la representación parlamentaria, ya que su triunfo quedaba asegurado en esos departamentos 5 .

4 En diversos incisos del artículo 11 de la Constitución de 1830 se establece la suspensión de la ciudadanía, entre otras causales, "por la condición de sirviente a sueldo, peón jornalero, simple soldado de línea, notoriamente vago (...) el hábito de ebriedad (...) no saber leer ni escribir... "y por tener deudas de diversa índole.

5 Como sostiene Pivel Devoto (1994. P. 228), el “...gobierno del departamento garantizaba al partido que 
Sin embargo, este tipo de acuerdo, informal y relativamente precario, no resolvía el conjunto de la conflictividad política. En primer lugar, porque se trataba de un acuerdo que congelaba la relación de fuerzas entre los partidos y eso lo convertía en crónicamente inestable. Desde 1865 el Partido Colorado se había establecido en el poder, como resultado de la revolución encabezada por Venancio Flores, y seguiría usufructuando esa posición de privilegio durante casi un siglo, con la única excepción, quizá, del breve gobierno de Latorre (1876-1880). En esas condiciones, el reparto de coparticipación era fuertemente asimétrico y no proporcionaba a los pactantes garantías en el largo plazo.

En segundo lugar, el acuerdo de coparticipación no podía ser estable porque no permitía dar cuenta de la diversidad interna de los partidos tradicionales. En torno a 1872, Pivel Devoto (1994, p. 234) detecta tres tendencias dentro del Partido Colorado: "los «ultras» de Francisco Bauzá, los «netos» de José Cándido Bustamante y los conservadores o liberales de José P. Ramirez" y dos corrientes dentro de los blancos: "... la tendencia popular y caudillista del partido blanco y el grupo principista cuyo expositor doctrinario era Agustín de Vedia” (1994, p. 237).

En esos años comenzaron a funcionar los "clubes" políticos, primeros antecedentes orgánicos de los partidos políticos en un sentido moderno del término, con programas, directivas y estatutos formalmente adoptados. Los primeros en fundarse fueron el Club Radical -principistas que renegaban de las divisas tradicionales- de muy efímera trayectoria, el Club Libertad -que en un principio pretendió unificar a los colorados principistas (ex-conservadores) y a los "netos" (caudillistas ex"floristas")- y el Club Nacional -blancos principistas- que daría la nueva y definitiva denominación a los blancos. Este período de conflictos cruzados, inter e intrapartidarios condujo rápidamente a la década del "militarismo" (1876-1886) en la que la vida política partidaria estuvo más bien silenciada.

Simultáneamente, el período entre 1872 y 1897 se caracteriza por dar inicio al debate en torno a la representación de minorías y la representación proporcional, la propuesta del uso del doble voto simultáneo y por la presentación de proyectos en esa dirección, aunque hasta 1898 ninguno tuvo andamiento. Precisamente, a comienzos de dicho período se incorpora por primera vez en los programas de los partidos políticos el reclamo de la representación proporcional ${ }^{6}$.

En 1876, Justino Jiménez de Aréchaga emerge como uno de los protagonistas fundamentales en el debate sobre las reglas electorales y la representación de las minorías mediante la presentación de un proyecto de ley en el que, como novedades, proponía la representación proporcional, el doble voto simultáneo y el reconocimiento a los partidos políticos. Dicho proyecto, que "...fue recibido con indiferencia cuando no combatido abiertamente..." (Pivel Devoto, 1994, p. 348), puede ubicarse

lo ejercía, elecciones libres [sic] en esa región y por lo tanto el triunfo en los comicios, lo cual significa la posesión total de las bancas que correspondía elegir en virtud del sistema electoral de mayoría, entonces vigente".

6 Según Pivel Devoto (1994, p. 332), el “...Partido Radical y el Partido Nacional [los clubes] al formular en 1872 su declaración de principios, proclamaron por vez primera en el pais lo que a partir de aquella fecha habria de convertirse en una bandera política: la idea de la representación proporcional en la elección de Representantes". 
como el primer antecedente del que sería, con el paso de los años, el peculiar sistema electoral uruguayo que rigió durante casi todo el siglo XX. Más tarde, Jiménez de Aréchaga, condensaría sus ideas sobre sistema electoral en "La Libertad Política", publicado en $1883^{7}$.

Durante el siglo XIX se desarrolló una profusa legislación en materia electoral, pero nunca se logró la voluntad suficiente para modificar los mecanismos de representación ${ }^{8}$. Sin embargo, los acontecimientos de 1897 tendrían una gravitación decisiva en la futura evolución institucional. Disconformes con la política "exclusivista" que habían llevado adelante los últimos gobernantes colorados, los blancos se levantaron una vez más en armas. Esta revolución, conducida por Aparicio Saravia, tuvo su fin en un acuerdo de paz que renovó y amplió las bases de la coparticipación entre blancos y colorados -adjudicándose a los primeros seis jefaturas departamentales- y logró la incorporación, por primera vez en la historia, de la representación de la minoría en las elecciones de Representantes.

La coyuntura de 1897, con la renovación del pacto de coparticipación, pero fundamentalmente, con la inclusión de la representación de las minorías a nivel legislativo, marca el inicio de un período que culminaría con la reforma constitucional de 1917 y que está signado por la búsqueda de un sistema electoral que articule los intereses de los dos partidos principales en base a las características recién descritas y permita su competencia pacífica y puramente electoral por el gobierno.

El 22 de octubre del año 1898 se consagra la ley acordada el año anterior en el "Pacto de la Cruz" (conocida como la "ley del tercio") por la que se asignaba la tercera parte de los cargos de diputado a la minoría de un departamento siempre que superara el cuarto de la votación en ese lugar. Sin embargo, esa ley no llegó a aplicarse porque blancos y colorados, mientras duró la precaria armonía, prefirieron realizar acuerdos en los que se repartían las bancas formando "listas mixtas". Luego de unos años de calma -en 1904- el entonces Presidente José Batlle y Ordoñez designa los Jefes Políticos de los departamentos blancos sin consultar a los dirigentes de esa colectividad, cuestión que fue suficiente para dar por violado el pacto de $1897 \mathrm{e}$ iniciar la última gran guerra civil en Uruguay. Batlle y Ordońez no era propenso a la coparticipación y, en particular, a que el país no tuviera un gobierno centralizado. Contando con un ejército mucho más numeroso que en el siglo XIX y mucho mejor armado, pudo controlar la sublevación que se desvaneció poco después de la muerte en combate de su líder, Aparicio Saravia. Poco después de la derrota de la revolución

\footnotetext{
7 Jiménez de Aréchaga (1883, p. 107) acusaba directamente al sistema electoral mayoritario de ser la causa de la inestabilidad política en Uruguay: "Nuestro sistema electoral es contrario a la paz pública y constituye una fuente permanente de anarquía y de despotismo, porque cada periodo electoral, los partidos politicos que se ven injustamente despojados de toda representación en el seno de los Poderes Públicos, no pudiendo conformarse con esa iniquidad, aunque ella proceda de la ley, se precipitan a recuperar sus derechos usurpados por medio de la sangre y el humo de los combates, ahondando asi cada vez más el abismo de las pasiones y de odios profundos que separa a los ciudadanos y que impide toda tentativa de concordia".

8 Pivel Devoto (1994, p. 485) sintetiza la evolución en la materia diciendo que desde “...1830 hasta 1893 la legislación electoral del pais habia progresado notablemente en lo que respecta a la organización del Registro Civico y a las disposiciones que debian regir para la emisión del sufragio, aun cuando con ello no se hubiese logrado destruir la práctica de la intervención oficial ni estimular la concurrencia del pueblo a las urnas".
} 
blanca, la primera ley que se dicta en materia electoral del 27 de diciembre de 1904, restringía la representación de la minoría, aunque no la eliminaba?.

Sin embargo, su sucesor, el Dr. Claudio Williman, buscó tranquilizar a sus rivales subsanando los aspectos conflictivos en el terreno electoral. Haciendo honor a su promesa "... promulgó las leyes electorales de setiembre 2 de 1907, y julio 11 de 1910, que corrigen algunos de los más graves defectos de la "ley del mal tercio" de 1904" (Pérez y Castellanos, 1980 p. 209) ${ }^{10}$. Particularmente la segunda de ellas "...amplió y mejoró las bases generales de la representación legislativa mediante el aumento de bancas (90 en total), y el cambio del sistema electoral, mediante un complicado sistema de fracciones de unidad (cuartos, tercios, avos) que mejoraba la situación del partido minoritario" (Pérez y Castellanos, 1980, p. 209). Sin embargo, la patología política nacional, abstención y conspiración, se reprodujo mostrando la insuficiencia y disfuncionalidad de todos los mecanismos implementados. Y no se puede decir que se tratara de métodos excluyentes; por ejemplo, la elección de 1910, con su sistema de representación de minorías, incorporó al parlamento al Partido Socialista y al Club Liberal que, concurriendo aliados a los comicios, ocuparon, con una exigua votación, dos bancas reservadas a la minoría, que fueron posibles gracias a la abstención del Partido Nacional en rechazo a la proclamación de la candidatura presidencial de José Batlle y Ordóńez. La ley de 1910 también incorporó, por vez primera, el doble voto simultáneo, otro instrumento cuya finalidad era permitir la pugna interna dentro de los partidos sin necesidad de que se produjeran divisiones. Este instrumento, que hubiera sido promocionado, como ya se dijo, por Justino Jiménez de Aréchaga en el siglo XIX, pasaría a formar parte, desde entonces, de los rasgos configuradores del sistema electoral uruguayo.

Pero hasta entonces el sistema no se encontraba en equilibrio o, en todo caso, generaba un equilibrio inestable: el Partido Colorado abusaba de su posición dominante y el Partido Nacional se rebelaba acudiendo a las armas. Como equilibrio, esta situación tiene características similares a las de un dilema del prisionero, donde los jugadores utilizan la estrategia que les asegura mayores ganancias (la "traición") pero terminan obteniendo menos que lo que obtendrían si ambos usaran la estrategia alternativa: la cooperación. La solución del conflicto a través de un pacto constitucional permite resolver el problema modificando los incentivos de los actores.

9 Según Pérez y Castellanos (1980, p. 204): "Por dicha ley -que la minoría «nacionalista" perjudicada, llamó del «mal tercio»-ésta obtendría representación si lograba la tercera parte de los votos emitidos, en lugar del cuarto como se exigía antes; ello ocurría en sólo siete departamentos cuyo número de bancas legislativas era divisible por tres, de acuerdo a la nueva ley".

$10 \mathrm{Al}$ prestar juramento afirmó: "Los partidos pueden estar seguros de que gozarán de la misma amplia y absoluta libertad electoral y de que, observando la debida imparcialidad, rodearé de todas las garantías el sufragio y haré respetar su resultado" (citado en Acevedo, 1943, p. 267). 


\section{Hacia la Constituyente}

Los hechos ocurridos durante la primera década del siglo XX mostraron que la democratización del país requería una gran operación de ingeniería constitucional, ya que las concesiones puntuales no lograban que la oposición legitimara al gobierno ${ }^{11}$. Precisamente, la elaboración de un marco institucional democrático consistió en un conflictivo proceso que, a lo largo de una década, intentó articular de forma equilibrada los intereses de los dos principales partidos políticos. La activación del proceso de reforma constitucional, realizada a fines de 1907, iba a marcar la escena política de los años siguientes, hasta la elección de una Convención Nacional Constituyente en 1916.

Sin embargo, el debate a partir de 1913, no giró ya en torno a la representación proporcional y las garantías del sufragio, sino que se centró en la conformación del Poder Ejecutivo. El Presidente Batlle, en ejercicio de su segundo mandato (19111915) había publicitado a través de la prensa su intención de crear un órgano colegiado que cumpliera las funciones del Ejecutivo. Esta propuesta motivó el rechazo, no sólo del Partido Nacional, sino de un grupo conservador del Partido Colorado, que se definió como anticolegialista -luego autodenominado Partido Colorado General Fructuoso Rivera- y que fuera conocido de ahí en más como "riverismo". El riverismo, actor clave en este proceso, se oponía a la continuidad del reformismo batllista por su carácter intervencionista y veía en el proyecto colegialista, al igual que los blancos, una maniobra para continuar en el poder.

La Constitución de 1830 preveía un complejo mecanismo para su reforma "..., que exigia la previa declaración de la necesidad de la revisión constitucional por el Parlamento y luego la aprobación de la o de las reformas por dos legislaturas sucesivas" (Gros Espiell y Arteaga, 1991, p. 59). Lo que se reformó siguiendo ese procedimiento -luego de aprobar con ese fin leyes en 1907, 1910 y, finalmente, en 1912-, fue el propio mecanismo de reforma constitucional, que ahora "...exigía la previa declaración de la conveniencia nacional de la reforma por las dos terceras partes de votos de ambas Cámaras, producido lo cual, se convocaría a una Convención Nacional Constituyente, que debia estudiar las enmiendas, las cuales, si resultaren aprobadas por mayoría absoluta de votos, se someterían a la aprobación del Cuerpo Electoral" (Gros Espiell y Arteaga, 1991, p 59). Por ley del $1^{\circ}$ de setiembre de 1915 se convoca a la elección de una Convención Nacional Constituyente para el 30 de julio de 1916, que establecía por primera vez el voto secreto, el sufragio universal masculino y una forma limitada de representación proporcional. La Constituyente sesionó durante un año entre octubre de 1916 y octubre de 1917 y el texto sancionado fue sometido a ratificación plebiscitaria y promulgado el ańo siguiente.

En las elecciones para la Convención Nacional Constituyente del 30 de julio de 1916, el mapa político que daría el primer ordenamiento democrático al país

11 No en vano el acuerdo de paz que dio fin a la última revolución blanca estableció que: “...el Gobierno incluirá entre los asuntos de la convocatoria extraordinaria la reforma de la Constitución, quedando el Poder Legislativo en completa libertad para decretarla o no y sancionar en el primer caso las reformas que juzgue convenientes..." (citado en Acevedo 1943, p. 265). 
estaba constituido por el tradicional bipartidismo, pero con una peculiaridad: en esos comicios, que bien pueden calificarse como las primeras elecciones legítimas de la historia uruguaya, el oficialismo fue derrotado ${ }^{12}$. Aunque el Partido Colorado contaba con la mayoría, la disidencia del riverismo, dejó al batllismo y a su proyecto colegialista en minoría, haciendo imprescindible la negociación. El proceso de negociación de la Constitución se desarrolló, consecuentemente, en el marco de un equilibrio inestable, con una Asamblea Constituyente hostil al gobierno y un Gobierno con poderes suficientes para bloquear el proceso reformista. Plausiblemente, fue por ese peculiar balance de fuerzas que la Constituyente pudo terminar elaborando un proyecto consensuado, producto de un pacto entre el nacionalismo y el batllismo que implicó concesiones recíprocas.

Las características del sistema político uruguayo del momento, fundamentalmente su persistente bipartidismo fraccionalizado, en el contexto del peculiar balance de fuerzas que se había creado en el momento, hicieron necesaria la adopción de un conjunto de instrumentos que lograran el consenso de los pactantes y que establecieran la norma del consenso como la única que permite establecer las reglas de juego. En esas condiciones, la interacción entre los jugadores ofrece incentivos para la coordinación y la estrategia cooperativa puede resultar la mejor estrategia para que todos puedan lograr, si no su mejor opción, al menos la segunda. La inclusión de una serie de instrumentos (representación proporcional legislativa, elección directa y por mayoría relativa del Ejecutivo y doble voto simultáneo) en el ordenamiento jurídico-electoral uruguayo, busca articular las preferencias de los actores políticos más relevantes para lograr un amplio acuerdo. El nacimiento de la democracia uruguaya se basó en un amplio consenso y el mantenimiento del consenso se convirtió en un requisito para su continuidad (Chasquetti y Buquet, 2004; Pérez Antón, 1988).

Para los colorados, instalados en el gobierno y el poder de la maquinaria estatal, la instrumentación de un régimen democrático legítimo se tornaba la vía menos costosa de ejercer el gobierno. El riesgo de perderlo pasaba a ser un perjuicio menor comparado con el permanente cuestionamiento civil y armado al que estaban sometidos. Para los blancos, las elecciones democráticas representaban no sólo el menos costoso de los medios de alcanzar el gobierno, sino probablemente el único. La pacificación requería un compromiso que involucrara a blancos y colorados, no podía ser impuesta desde el poder, como lo demostró la evolución política entre 1872 y 1915 . Pero ese compromiso no podía ser un mero reparto de posiciones de poder, sino el establecimiento de reglas democráticas de competencia y distribución del gobierno que articularan adecuadamente sus intereses (Pareja, 1988) ${ }^{13}$.

12 "Los nacionalistas obtuvieron 105 convencionales, los colorados colegialistas 87, los colorados anticolegialistas 22, los socialistas 2, los católicos 2" (Acevedo, 1943, p. 288).

13 Así surge claramente del informe que fundamentaba el acuerdo entre el batllismo y el nacionalismo en la Convención Nacional Constituyente de 1917: "Este proyecto es el fruto de un acuerdo constitucional efectuado por los representantes de las dos mayores fuerzas politicas del país, [...] la aprobación de este avenimiento, primero por Vuestra Honorabilidad y luego por el pueblo, en la ratificación plebiscitaria lleva consigo la solución de una de nuestras crisis más graves, asegurando el bienestar y la paz, a cuyo amparo y bajo el influjo de sanos principios democráticos, podrá el país imponer sus designios y reparar, por medio de la tranquilidad y el trabajo, el desgaste de una recia y continuada lucha política.... la ley fundamental de un pueblo, que regirá para el 
El conjunto de normas establecidas en la nueva Constitución, no sólo facilitó el surgimiento de un régimen político democrático, sino que generó las condiciones para su estabilidad y su futura adaptación, en la medida en que el consenso no sólo se logró para esa ocasión, sino que también se transformó en exigencia, de ahí en más, como sustento de la convivencia pacífica, impidiendo que las reglas de juego se modifiquen sin un amplio acuerdo interpartidario. Para asegurar el concurso de los dos principales partidos en las sucesivas modificaciones a la normativa electoral o, por la negativa, para asegurarle a cada uno de ellos el derecho de veto, se articulan dos disposiciones que resultan mutuamente imprescindibles: por un lado, la representación proporcional a nivel legislativo y, por otro, la exigencia de dos tercios de legisladores para modificar las disposiciones electorales.

Cuando en 1917 se formuló el acuerdo electoral, la representación proporcional y la exigencia de dos tercios de legisladores para su modificación quedaron unidas en una única formulación ${ }^{14}$. En consecuencia, el único escenario posible para la modificación de la representación proporcional y de las demás disposiciones electorales, pasó a ser un acuerdo entre los dos partidos principales o, por lo menos, de la mayoría de ambos. El consenso, que a todas luces aparecía como una necesidad funcional del sistema político, se tradujo en norma constitucional ${ }^{15}$.

Aunque un sistema mayoritario le asegura mayorías legislativas al gobierno y permite la representación parlamentaria a la oposición, puede dejar afuera o sumamente sub-representadas a las fracciones minoritarias de ambos partidos o, alternativamente, esas fracciones minoritarias estarían impedidas de competir electoralmente y serían obligadas a negociar y someterse a la mayoría partidaria. Consecuentemente, es de esperar que fueran precisamente las fracciones minoritarias de los partidos tradicionales quienes abogaran por la representación proporcional. La posición de los

presente y para el futuro, debe reunir el mayor número de sufragios para que sea prestigiosa y eficaz". Tomado del Informe de la Comisión de Constitución presentado en la 54a. Sesión ordinaria de la Convención Nacional Constituyente del 25 de junio de 1917, Diario de Sesiones, Tomo III, pág. 26. En adelante las referencias al Diario de Sesiones de la Convención Nacional Constituyente de 1916-1917 se mencionarán como $\mathrm{CNC}$, seguidas del tomo y el número de página.

14 En el artículo 46, incluido en las disposiciones transitorias del Proyecto de Reforma Constitucional presentado en la 51a. sesión ordinaria del 6 de junio de 1917, se estableció:

"Para las elecciones a las que se refiere el artículo anterior (después del $1^{\circ}$ de marzo de 1919), regirán las disposiciones de la ley de $1^{\circ}$ de setiembre de 1915 y complementarias dictadas hasta el 30 de julio de 1916, así como las vigentes en la actualidad sobre el número de diputados por Departamento, en tanto no se reformen esas leyes por dos tercios de votos de cada Cámara. No son aplicables a la distribución de Diputados u otros cargos electivos, las disposiciones de la ley de $1^{\circ}$ de setiembre de 1915 que atribuyen a la mayoría los tres quintos. La repartición se hará por el cociente electoral". (CNC, III, p. 8).

15 Así lo reconocía a título expreso el constituyente Martín C. Martínez: “...obtenemos también garantías de estabilidad para las leyes electorales, las que ya no podrán retocarse más en visperas de comicios, según los intereses y las pasiones de partido, sino que los dos partidos deberán ponerse de acuerdo para modificar la legislación vigente el 30 de julio, que era bastante aceptable" (CNC, III, p. 247). Coincidente era la opinión del constituyente Washington Beltrán: "Establecemos que para la reforma de la Constitución se necesitan las dos terceras partes de votos; incorporamos lo que siempre hemos sostenido: que la Constitución, por ser ley fundamental de carácter permanente, no debe ser obra de un partido, sino hecha por los orientales para los orientales" (CNC, III, p. 359). 
disidentes colorados a favor de la proporcionalidad, además de justificada, era decisiva, ya que sus votos sumados a los nacionalistas eran mayoría en la Convención ${ }^{16}$.

Por otra parte, la representación proporcional permite, dentro de un formato bipartidista, la presencia mayoritaria del partido ganador de las elecciones en el Parlamento, pero, en contraposición, la fraccionalización interna puede dificultar la coherencia de gobierno que reclamaban los colorados. Por esta razón, las modificaciones al régimen de representación tuvieron fuertes resistencias por parte del batllismo, que veía en la representación proporcional el peligro de fomentar las ya importantes disidencias internas existentes. Era necesario instrumentar una instancia institucional que garantizara la unidad de los partidos en el ejercicio del gobierno, para poder finalmente aceptar la demanda nacionalista y riverista de proporcionalidad "integral". El armado jurídico-institucional debía, entonces, encontrar el equilibrio que resolviera la contradicción.

Apenas comenzadas las sesiones, la Corporación de Constituyentes Nacionalistas presentó su proyecto de Constitución que establecía que el “... Presidente y Vicepresidente de la República serán elegidos por un Congreso de Electores, que el pueblo nombrará por el sistema de la representación proporcional, voto secreto y obligatorio y demás garantías..." (CNC, I, p. 186). En la lógica de un sistema institucional de este tipo, los colorados veían la posibilidad de que se pudiera nominar a los integrantes del Poder Ejecutivo a través de coaliciones poselectorales, haciendo de la representación proporcional un fuerte estímulo a la división interna de los partidos, que el oficialismo deseaba evitar a toda costa. Esta propuesta del nacionalismo premiaría a las minorías disidentes, dándoles la oportunidad de ser decisivas a la hora de elegir a los integrantes del Poder Ejecutivo, por lo menos en caso de una elección reñida en las urnas.

Así fue que el batllismo se opuso, no a la representación proporcional, sino a que fuera a partir de ella que resultara la designación del Poder Ejecutivo. Se podía aceptar la representación proporcional siempre y cuando, como compensación, existiera una instancia mayoritaria para la formación del gobierno ${ }^{17}$. El mecanismo que se encontró fue la elección directa, por mayoría relativa y con doble voto simultáneo del Presidente de la República. El doble voto simultáneo estimulaba la permanencia de eventuales disidentes dentro del partido, ya que votar por fuera de los grandes

16 Pedro Manini Ríos, líder del riverismo, fracción minoritaria del Partido Colorado y única fracción minoritaria orgánicamente presente en la Constituyente decía que la representación proporcional integral "...figura en el programa del Partido Colorado del año 1907, aquel programa común que a todos nos unía, que no dividia a nuestro partido, y que es, precisamente, con algunas variaciones y adiciones, el que sostiene en la actualidad como bandera de principios, el grupo colorado riverista". (CNC, II, p. 212).

17 La posición del batllismo está claramente expresada en las siguientes declaraciones del constituyente Julio María Sosa: "Yo soy miembro de un partido que ha proclamado hace ya muchos años, cuando todavía los otros partidos no pensaban en estas avanzadas normas democráticas, el principio de la representación proporcional [...] Pero no la ha realizado [...] porque en el programa de mi partido [...] se establece la expresa salvedad de que no se consignará en las leyes electorales el principio de la representación proporcional, mientras a las Cámaras Legislativas no se les quite la facultad de elegir Presidente de la República [...] si la Asamblea Legislativa eligiese Presidente, no debe a su vez ser elegida por el principio de la representación proporcional [...porque...] el Poder Ejecutivo debe ser sintesis del programa de un sólo partido de gobierno..." (CNC, II, p. 170). 
lemas pondría en tela de juicio las posibilidades de triunfo del propio partido. Pero, a la vez, permitía que quien ganara las elecciones tuviera la capacidad para conducir el gobierno. Adicionalmente, la creación del Concejo Nacional de Administración, con representación de la minoría, fue el necesario complemento de transacción con la fórmula colegialista.

El acuerdo logrado entre el nacionalismo y el oficialismo estipuló que el "... Presidente de la República será elegido directamente por el pueblo, a mayoría simple de votantes, mediante el sistema de doble voto simultáneo..." (CNC, III, p. 28) y el “... Consejo Nacional de Administración se compondrá de nueve miembros, elegidos directamente por el pueblo, mediante el sistema de doble voto simultáneo, por lista incompleta, [...] correspondiendo las dos terceras partes de la representación a la lista más votada, y la tercera parte restante a la del otro partido que le siga en el número de sufragios obtenidos..." (CNC, III, p. 30).

De esta forma, terminan de encajar todas las piezas del rompecabezas: por un lado, representación proporcional legislativa promovida por los nacionalistas y los riversistas, junto a la exigencia de dos tercios para modificar las reglas electorales y la propia Constitución y, por otro, elección directa por mayoría relativa y doble voto simultáneo para la elección de un Ejecutivo bicéfalo que contemplaba, al menos parcialmente, el proyecto colegialista del batllismo. La narrativa desarrollada hasta aquí ha detectado los componentes del diseño institucional que se articularon para satisfacer los intereses de los actores involucrados en el proceso y generar un resultado de equilibrio con capacidad de mantenerse en el tiempo. La siguiente sección presenta una modelación del proceso utilizando el instrumental de la teoría de juegos.

\section{El juego constitucional}

Ya hemos sostenido que el hecho de que los acuerdos de paz de 1872 y 1898 fueran informales, no era una condición suficiente ni necesaria para explicar la inestabilidad política observada durante casi todo el siglo XIX. Sin embargo, nuestra narrativa tampoco ofrece una respuesta precisa para explicar por qué los acuerdos entre los partidos eran inestables antes de 1916. Dicho de otro modo, hemos explorado las causas de la inestabilidad política durante el siglo XIX sin proveer una explicación de por qué los partidos alcanzaron un acuerdo para reformar la constitución en 1916. Este acuerdo, no solo sería de carácter formal, sino que, además, se constituiría en un equilibrio estable en la interacción entre los agentes. Hasta ahora, las causas del cambio constitucional y la estabilidad política después de 1916, aunque profusamente descritas, permanecen inexplicadas.

Precisamente, esta sección provee una explicación plausible mediante el desarrollo de un modelo de teoría de juegos, que no solo permite identificar la racionalidad y estrategias de los actores involucrados, sino fundamentalmente la naturaleza auto reforzada (self-enforced) del equilibrio alcanzado a través del nuevo texto constitucional. Dado que la mayoría de los casos de construcción institucional implican un proceso que no puede ser captado mediante un juego de una sola jugada (single- 
shot) como el dilema del prisionero, esta sección desarrolla una versión extendida de un juego que capta las estrategias y decisiones de los partidos desarrolladas en nuestra narrativa. Los juegos extendidos son más apropiados que el dilema del prisionero, donde la no-cooperación es la estrategia dominante para los agentes y la matriz de pagos no permite observar la dinámica de la interacción entre estos. Un juego extendido no solo permite explicar la cooperación, sino que además nos permite captar la forma en que cada jugador responde interactivamente a las acciones de otros jugadores a través del tiempo.

El equilibrio hasta 1916 puede ser fácilmente representado por un juego con dos jugadores $(\mathrm{C}=$ Colorado; $\mathrm{B}=\mathrm{Blanco})$ y dos posibles acciones para cada jugador, tal que:

$C=\{F=$ Fraude $; F=$ No - Fraude $\}$

$B=\{P=$ Participar,$N P=N o-$ Participar $($ elecciones $)\}$

Donde:

$\mathrm{G}=$ beneficio de ganar la elección o de obtener los cargos de gobierno. $\alpha=$ la probabilidad de ganar la elección o la proporción de cargos para C. $1-\alpha=$ la probabilidad de que B gane la elección o su proporción de cargos. $K=$ costos de legitimidad para los partidos cuando cometen fraude o cuando se someten al fraude o cuando se abstienen sin que haya fraude.

Asumiendo que el partido en el gobierno (C) mueve primero, la secuencia de movimientos puede ser representada en el Diagrama 1, incluida la estructura de pagos para los jugadores:

\section{Diagrama 1. El equilibro decimonónico}

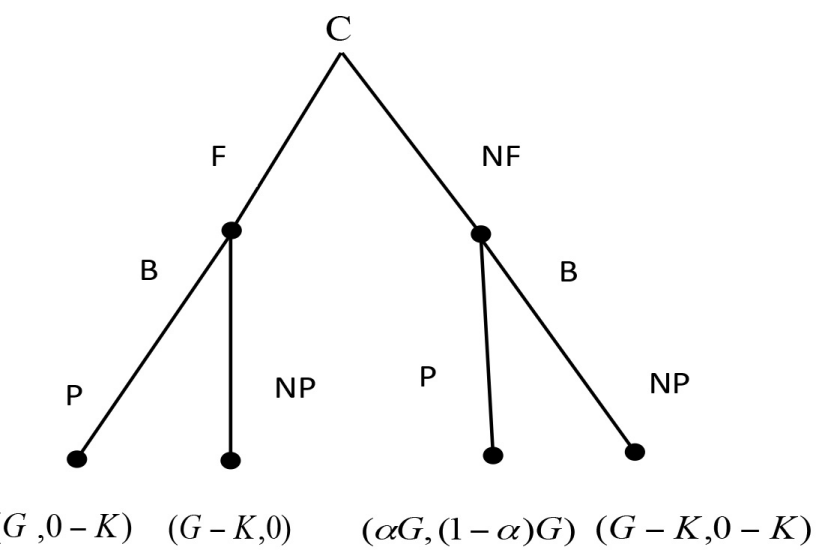

Fuente: Elaboración propia. 
Cuando C juega F y B juega $\mathrm{P},(\mathrm{G})$ y $(0-K)$ son los pagos para C y B respectivamente. Nótese que aquí asumimos que la probabilidad de ganar la elección para C es 1 cuando hay fraude $(\alpha=1)$ y, por lo tanto, obtiene la totalidad del beneficio que proporciona ocupar el gobierno. En este camino, C está dispuesto a pagar el costo de cometer fraude $(K)$ pero si B participa no lo paga porque su participación lo legitima. Para B, dado que $1-\alpha=0$ (su probabilidad de ganar la elección o su participación en el gobierno es nula), no hay beneficio y también debe pagar el costo de legitimidad que le genera haber convalidado una elección fraudulenta. Por otra parte, cuando $\mathrm{C}$ ha decidido cometer fraude, la alternativa para $\mathrm{B}$ es abstenerse (NP). En este caso $\alpha=1$ también, pero la utilidad de $\mathrm{C}$ disminuye porque enfrenta el costo del fraude $(K)$ mientras $\mathrm{B}$ no obtiene ningún beneficio, pero tampoco paga costos al abstenerse de participar en la elección.

Cuando $\mathrm{C}$ juega (NF) y B juega $\mathrm{P}$, la utilidad para $\mathrm{C}$ es $\alpha G$, mientras que $\mathrm{B}$ recibirá $(1-\alpha) G$. En este caso, alfa es menor que 1 , dado que existe una probabilidad positiva de que $\mathrm{C}$ pierda la elección o de que no se quede con la totalidad de los cargos de gobierno. En la misma línea, $1-\alpha$ es mayor que 0 , revelando que $\mathrm{B}$ podría ganar la elección u obtener una parte de los cargos de gobierno. Finalmente, cuando las jugadas son NF y NP, la probabilidad de ganar la elección para $\mathrm{C}$ vuelve a ser igual 1, dado que $\mathrm{B}$ se abstiene. Pero en este caso $\mathrm{C}$ debe pagar el costo de legitimidad por participar solo en la elección y B también paga el costo por no participar en una elección no fraudulenta.

Usando inducción retrospectiva para resolver el juego, luego de que $\mathrm{C}$ mueve (en el segundo estadio del juego), si B se encuentra en el nodo $\mathrm{F}$, jugará NP porque $0>-K$. En cambio, si $\mathrm{B}$ se encuentra en el nodo NF jugará $\mathrm{P}$, dado que $(1-\alpha) G>0-K$. Ahora, (en el primer estadio del juego), dado que $\mathrm{C}$ conoce la estrategia óptima de B, compara los pagos de los perfiles de estrategia (F, NP) y (NF, P). Ya sabemos por la narrativa que el equilibrio hasta 1916 era (F, NP) y por tanto deducimos que $G-K$ debería haber sido mayor que $\alpha G$. Esto es, los costos asociados al fraude electoral y la abstención de $\mathrm{B}$, no serían tan grandes como la pérdida que implica la disminución en la probabilidad de ganar la elección $1-\alpha$. Dicho de otro modo, los colorados prefieren pagar el costo de legitimidad de incurrir en fraude electoral, antes que arriesgar una derrota electoral o compartir el gobierno. Pero este equilibrio no puede ser estable porque los blancos no obtienen nada, lo que conduce reiteradamente a la configuración del conflicto bélico. Este parece ser el equilibrio hasta 1916, donde los colorados incurrían en fraude electoral, forzando a los blancos a no participar e inducirlos así a ingresar en un antebelum reiterado durante la mayor parte del siglo XIX.

Hasta 1904, los acuerdos de paz firmados en 1872 y 1898 implicaron una división del país sobre bases geográficas o lo que comúnmente llamamos arreglos de coparticipación. Sin embargo, la guerra civil de 1904 produjo un ganador dispuesto a no seguir el camino de los acuerdos de coparticipación, dejando a su rival con las manos vacías. Como vimos en la narrativa, la derrota del Partido Nacional dejó nuevamente al Partido Colorado ante dos alternativas en su condición de partido hegemónico: permanecer en esta condición a riesgo de enfrentar nuevamente una guerra 
y problemas de legitimidad constantes, o iniciar el camino de una reforma constitucional, contemplando objetivos ya explicitados en el Pacto de la Cruz de 1897 y la Paz de Aceguá de 1904 (Vázquez Romero y Reyes Abadie, 1986). Como vimos más arriba, el Partido Colorado inició en 1907 el proceso de reforma constitucional.

En 1916 el Poder Ejecutivo convocó a las elecciones para la Convención Nacional Constituyente, previstas por la ley de setiembre de 1915. Una parte del proyecto original de Batlle contemplaba las demandas históricas de los blancos, como la representación proporcional y los derechos asociados al voto, pero su interés primario finalmente se centró en el Ejecutivo Colegiado y su elección directa por doble voto simultáneo. Como vimos, el batllismo perdió la elección para la Constituyente ante la oposición de blancos y riveristas. Esta fracción dentro del Partido Colorado se tornó en un agente pivotal en el proceso de negociación de la reforma, no solo por oponerse fuertemente al proyecto de Ejecutivo colegiado de Batlle, sino por su orientación fuertemente conservadora y opositora de la agenda reformista del segundo gobierno del presidente Batlle. Esta división dentro del Partido Colorado es fundamental para poder explicar el acuerdo logrado en 1916.

Nuestro primer juego mostró que el equilibrio decimonónico, marcado por el fraude y la abstención, dominó la escena política durante el siglo XIX. La propuesta de reforma constitucional de Batlle implicó la transición hacia un nuevo equilibrio donde el fraude sería reemplazado por elecciones limpias y justas, en las cuales los blancos tendrían un claro incentivo a competir. Con la activación de los mecanismos para iniciar el proceso de reforma constitucional, los partidos comenzaron lentamente a jugar el juego democrático. Sin embargo, todavía no explicamos por qué los agentes acuerdan una reforma. Ex ante, para los colorados se había tornado insostenible la condición de inestabilidad y deslegitimidad del gobierno. Para los blancos o la oposición, era insostenible la falta de representatividad y transparencia en el proceso electoral. Ambos deseaban una reforma y el primer paso, formalmente, lo debía dar el gobierno de Batlle. 


\section{Diagrama 2. La reforma constitucional}

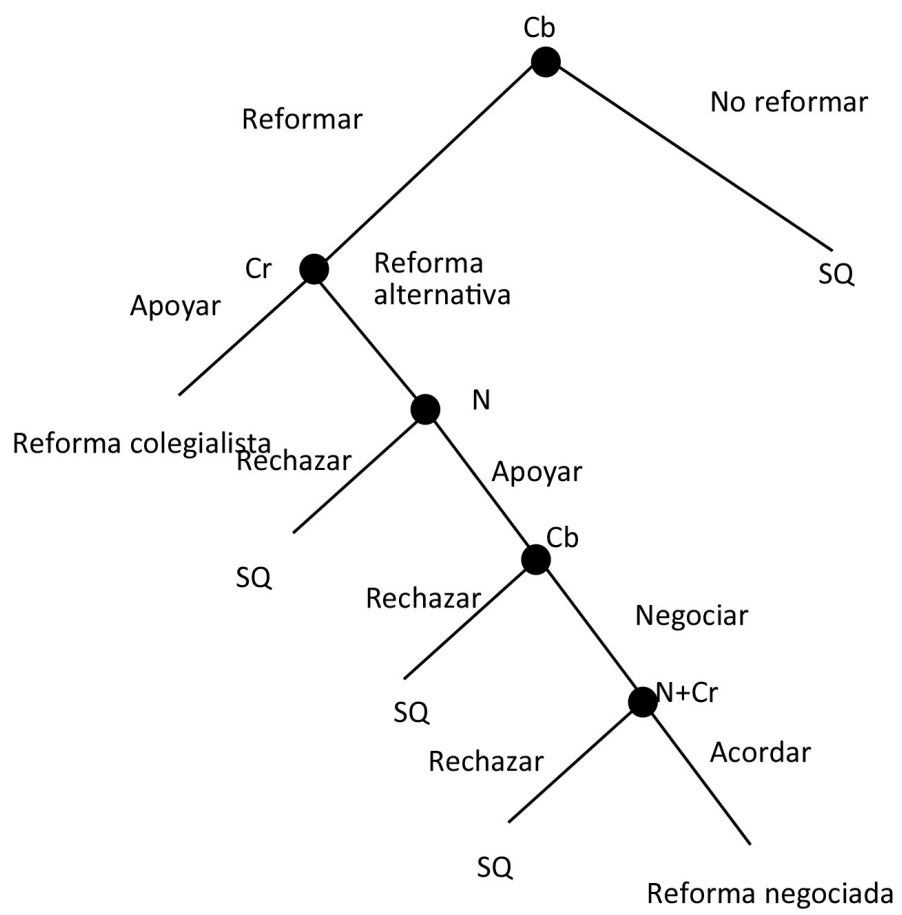

Fuente: Elaboración propia.

El juego del Diagrama 2, se inicia con la decisión del gobierno de Batlle de introducir una propuesta de reforma constitucional. La opción del status quo hubiera significado mantener el equilibrio inestable de nuestro primer juego, donde los blancos volverían a deslegitimar al gobierno, alzándose en armas contra el fraude y el exclusionismo anti-coparticipativo de Batlle ${ }^{18}$. Sin embargo, ante una propuesta de reforma (colegialista), el riverismo (dentro del Partido Colorado) se vio enfrentado a aceptarla o promover una reforma alternativa. Como vimos, el riverismo no estaba dispuesto a aceptar la propuesta original de Batlle, que no le ofrecía mayores ventajas respecto del statu quo de la constitución de 1830. Sin embargo, dado que ese equilibrio inestable era tan costoso como la reforma propuesta por Batlle, el riverismo tenía incentivos para proponer una reforma alternativa. Esta opción fue apoyada por los blancos, derivando en una nueva configuración en la que Batlle debía rechazar o proponer una nueva salida negociada en la que blancos y riveristas acordaran con el gobierno. Efectivamente, el último nodo de nuestro juego marca una configuración en la que blancos y riveristas acuerdan un nuevo texto constitucional con el gobierno de Batlle.

18 De hecho, en 1910 se reitera la configuración del antebellum típico que vimos durante todo el siglo XIX. 
En este modelo se introduce un nuevo jugador surgido de la división del Partido Colorado, por lo que representamos como $\mathrm{Cb}$ a la fracción batllista y como $\mathrm{Cr}$ a la fracción riverista. Además, se define una reforma como un cambio de un nuevo parámetro $\beta$ que representa la probabilidad de ganar la elección o la proporción de cargos para $\mathrm{Cb}$, por lo que $\alpha-\beta$ representa la probabilidad de que $\mathrm{Cr}$ gane la elección o su proporción de cargos, asumiendo que $\alpha \geq \beta$. Por un lado, este parámetro permite diferenciar, dentro del Partido Colorado, la parte de gobierno que le corresponde al batllismo y, por otro, la posible reducción de esa porción, como consecuencia de alguna reforma que conduzca a elecciones libres y justas. En el contexto del status quo, las utilidades en equilibrio de batillistas y riveristas serían $\beta G-K,(\alpha-\beta)(G-K)$ respectivamente, con $\alpha=1$. La reforma de la constitución modificaría el parámetro $\beta$ de forma que para la reforma colegialista tendríamos $\beta^{\prime}$ y para la reforma acordada $\beta^{\prime \prime}$ 'asumiendo que $\beta \geq \beta^{\prime}>\beta^{\prime \prime}$. Así, las utilidades de batllistas y riveristas con la reforma colegialista serían $\beta^{\prime} G,\left(\alpha-\beta^{\prime}\right) G$ y con la reforma acordada serían $\beta^{\prime \prime} G,\left(\alpha-\beta^{\prime \prime}\right) G$ respectivamente, pero ahora con $\alpha<1$. Por su parte, las utilidades de los nacionalistas serían 0 , tanto en el status quo como en la reforma colegialista ${ }^{19}$ y al menos $(1-\alpha) G$ con la reforma acordada, ya que como mínimo existirían elecciones sin fraude y, adicionalmente, podrían obtener otros beneficios.

Nuevamente usando inducción retrospectiva, cuando nos ubicamos en el último nodo del juego, vemos que tanto nacionalistas como riveristas preferirán la reforma acordada antes que el status quo porque $(1-\alpha) G>0$ y $\left(\alpha-\beta^{\prime \prime}\right) G>(\alpha-\beta)(G-K)$, es decir, los nacionalistas y los riveristas reciben una utilidad positiva de las elecciones sin fraude, que es mayor a no recibir nada con la situación del status quo para los nacionalistas y mayor también para los riversitas porque se reduce el peso del batllismo en el PC y se eliminan los costos que paga todo el partido en el status quo. Subiendo un nivel en el juego, los batllistas preferirán negociar siempre que $\beta^{\prime \prime} G>\beta G-K$, esto es, cuando sus beneficios por la porción de gobierno que les corresponde, excedan al beneficio de una porción mayor pero que implica costos. Sabemos por la narrativa que lo hicieron, por lo que podemos suponer que la diferencia entre $\beta^{\prime \prime}$ y $\beta$ era percibida como poco significativa y/o que el valor de $K$ era superior que en el pasado, algo que asumimos en la narrativa. Al llegar a este punto, entonces, los tres actores relevantes tienen incentivos para acordar, pero, ¿cómo se llegó hasta aquí? Tenemos que observar el paso anterior del juego, donde los nacionalistas, conociendo las mejores jugadas posteriores decidirán apoyar la reforma alternativa que promueven los riverisatas, simplemente porque nuevamente $(1-\alpha) G>0$, es decir, la posibilidad de ganar algo es mejor que el status quo en el que los nacionalistas no ganan nada. Del mismo modo, en la jugada anterior, los riveristas preferirán proponer una reforma alternativa porque por definición $\beta^{\prime}>\beta^{\prime \prime}$, es decir, una reforma alternativa disminuye el peso del batllismo en el gobierno, con respecto a la propuesta colegialista original.

19 En principio porque mantendrían la abstención, pero también porque su participación en esas condiciones no les daría espacio en el gobierno. 
Para culminar el razonamiento falta sólo explicar el principio del juego, la jugada del batllismo que decide iniciar el proceso reformista; ¿qué incentivos tenía para promoverlo? En condiciones de información completa y perfecta-que es lo que representa el modelo especificado- los batllistas preferirían iniciar el proceso reformista nuevamente si $\beta^{\prime \prime} G>\beta G-K$, lo que requiere los mismos supuestos mencionados antes, esto es, que los beneficios por la porción de gobierno surgida de elecciones sin fraude superen al beneficio de una porción mayor pero que implica costos de legitimidad. Pero es más razonable asumir la validez de este cálculo en el contexto de la negociación dentro de la Constituyente, que suponer que pudieron hacerlo varios años antes, ya sea en 1907 cuando se inició el proceso reformista sin contenidos específicos o en 1913, cuando Batlle promueve su propuesta colegialista. Aunque el resultado es el mismo, tal vez sea más razonable suponer que en el inicio del juego los parámetros no eran conocidos, ya que ocurrió antes de que se develara la correlación de fuerzas que surgió de la elección de constituyente ${ }^{20}$ y que, por lo tanto, se esperaba que el proceso condujera a una reforma colegialista donde $\beta^{\prime} G>\beta G-K$, es decir, el batllismo aceptaba resignar algún espacio de poder a través de elecciones libres y justas, pero manteniendo su hegemonía y eliminando los costos de legitimidad que le generaba el fraude y la abstención del nacionalismo.

Volviendo a la narrativa, es evidente que el presidente Batlle estaba decidido a avanzar con su propuesta de reforma y que, si bien su proyecto original de Ejecutivo colegiado había sido vetado por blancos y riveristas, los objetivos de reforma de la oposición eran viables siempre y cuando el nuevo Ejecutivo colegiado fuera electo con las reglas propuestas por el gobierno. Para la oposición de blancos y riveristas, el Ejecutivo colegiado por si solo era inviable como propuesta de reforma, siendo preferible el status quo antes que el proyecto original. Sin embargo, el proyecto negociado contempla largamente los objetivos de la oposición y abre el camino para la aceptación del gobierno (Martin C. Martínez, 1964).

\section{Conclusiones}

El pacto fundacional de la democracia uruguaya contiene una serie de arreglos constitucionales que han corrido variada suerte en el transcurso de un siglo de historia. De todos ellos, el único que a priori puede calificarse de imprescindible para la instauración de una democracia es el conjunto de garantías que se otorgan para el libre ejercicio del sufragio. No es posible construir democracia sin asegurar el voto secreto, igualitario y universal, así como la libertad política de partidos y electores, y el establecimiento de mecanismos de contralor electoral. Todos los demás aspectos

20 Se puede asumir que la norma de la ley de 1915, que asignaba la mayoría de los cargos de la Constituyente al partido que superara los dos quintos de los votos, buscaba asegurar la mayoría del batllismo en la Constituyente. Entonces podemos suponer también que, aún en 1915, el batllismo esperaba votar por encima de los dos quintos y tener mayoría en la Constituyente. Con más razón entonces, se puede asumir que el batllismo esperaba controlar el proceso constituyente en 1907 y también en 1913. 
contenidos en el pacto -los que propiamente pueden denominarse de ingeniería político-electoral-, y que fueron incorporados a la Constitución de 1918 (el Ejecutivo bicéfalo-semicolegiado, el doble voto simultáneo y la representación proporcional en la Cámara de Representantes) son contingentes.

Teórica y empíricamente, puede existir democracia con o sin el conjunto de arreglos constitucionales que formaron parte del pacto entre los partidos. Sin embargo, los arreglos constitucionales diseñados por la elite política uruguaya se constituyeron en una base fundamental para la construcción de una democracia a gran escala. Estos arreglos podrían no ser funcionales a la construcción de una democracia en otros casos, aun signados por conflictos políticos similares a los que Uruguay se vio enfrentado durante la mayor parte del siglo XIX (Schmitter y Karl, 1993; Hardin, 2008). Sin embargo, el singular conjunto de reglas y procedimientos que los partidos se dieron en la reforma constitucional de 1917, se constituyó en una configuración fundamental no solo para la estabilidad de corto plazo sino para el establecimiento de una democracia estable en el largo plazo.

La competencia por el poder existe en todas partes, pero puede tener diferentes formas. La lucha violenta por el poder sólo permite arribar a equilibrios sub-óptimos, como el dilema del prisionero. La superación de esta situación consiste en buscar reglas que favorezcan la cooperación entre los competidores, que les garanticen el acceso a la parte que le toca. Como sostiene Hardin (2008, pp. 291-2): “... las constituciones regulan la coordinación de la población en una forma de gobierno en particular y, por lo tanto, tienen la estructura estratégica de los juegos de coordinación. [...] En un juego de coordinación, el mejor resultado para cualquier jugador es también el mejor o casi el mejor para el otro jugador". La forma democrática de competir por el poder implica, además, que lo que le toca a cada uno se determina sobre la base del apoyo electoral. Pero como la competencia, por más libre y justa que sea, siempre determina ganadores y perdedores, el acuerdo no sólo requiere ponerse de acuerdo en reglas, sino mantener esas reglas a lo largo del tiempo, esto es, promover la cooperación intertemporal entre los actores ${ }^{21}$.

La reforma constitucional pudo haber fracasado. De hecho, nuestro análisis muestra que podría haber fracasado aun en presencia de preferencias normativas claramente orientadas hacia la democracia entre la elite reformadora. Este resultado era solo uno de los posibles equilibrios en el proceso de reforma, pero claramente no el único. Por tanto, la presencia de una elite con vocación o cultura democrática parece haber sido una condición necesaria pero no suficiente para la consecución de una reforma que hizo nacer a la democracia. Además, y fundamentalmente, el proceso de reforma se desarrolló en el marco de un duro conflicto político, no solo vinculado al diseño institucional que la Constituyente elaboraba. Por el contrario, una porción

21 Juan Andrés Ramírez (1967, p. 130), destacado líder de la oposición nacionalista y protagonista decisivo de aquel pacto fundacional, confirmará esta idea once años después: "Perder la elección significaba perderlo todo, y perderlo, según todas las probabilidades, sin más esperanza de recuperarlo que por medio de la violencia. Hoy el poder no está concentrado en ninguna parte. Todos los partidos saben que tendrán en él la parte a que su caudal político les da derecho. Saben que lo perdido en una elección puede ser recuperado en la siguiente, y que ya no hay ni habrá partidos ni ciudadanos extranjeros en el seno de la Patria". 
importante del conflicto entre los partidos y los grupos de presión de la época estaba teniendo lugar en el escenario general del sistema político, en el que el Alto de Viera había colocado la agenda reformista del batllismo en un impasse. Después de todo, el mismo Batlle que promovía una reforma constitucional indeseada por las fuerzas conservadoras, era quien lideraba una agenda reformista indeseada por esas mismas fuerzas y los grupos económicos de poder que las apoyaban. Irónicamente y contra algunas expectativas teóricas (Acemoglu y Robinson, 2006), esas fuerzas conservadoras contribuyeron decisivamente con la reforma constitucional que dio inicio al establecimiento de una democracia moderna.

\section{Bibliografía}

Acemoglu, Daron y James A. Robinson (2006). The Economic Origins of Dictatorship and Democracy. Cambridge, Cambridge University Press.

Acevedo, Eduardo (1942). Manual de Historia Uruguaya: Después de Artigas. Montevideo, A. Monteverde y Cía.

Almond, Gabriel y Sidney Verba (1963). The Civic Culture. Political Attitudes and Democracy in Five Nations. Newbury Park, Sage.

Bates, Robert H., Avner Greif, Margaret Levi, Jean-Laurent, Rosenthal, and Barry R. Weingast (1998). Analytic Narratives. Princeton, NJ: Princeton University Press.

Benoit, Kenneth (2004). "Models of electoral system change”, Electoral Studies, 23, pp. 363-389.

Boix, Carles (1999). "Setting the rules of the game: the choice of electoral systems in advanced democracies”, American Political Science Review, 93(3), pp. 609-624.

Caetano, Gerardo y José Rilla (1985). "El Sistema de Partidos: Raíces y Permanencias”, en Caetano, Gerardo, José Rilla, Pablo Mieres y Carlos Zubillaga: De la Tradición a la Crisis: Pasado y Presente de Nuestro Sistema de Partidos. Montevideo, CLAEH-Ediciones de la Banda Oriental.

Chaquetti, Daniel y Daniel Buquet (2004). "La democracia en Uruguay: una partidocracia de consenso". Política, 42, pp. 221-247.

Colomer, Josep (2005). "It's Parties that Choose Electoral Systems (or, Duverger's Laws Upside Down", Political Studies, 53, pp. 1-21.

Convención Nacional Constituyente (CNC 1925). Diario de Sesiones (3 tomos). Montevideo: Poder Legislativo.

Dahl, Robert A. (1971). Polyarchy: Participation and Opposition. New Haven: Yale University Press.

Gros Espiell, Héctor y Juan José Arteaga (1991). Esquema de la Evolución Constitucional del Uruguay. Montevideo: Fundación de Cultura Universitaria.

Hardin, Russell (2008). "Constitutionalism”, en Wittman Donald A. and Barry R. Weingast (eds.): The Oxford Handbook of Political Economy. Oxford: Oxford Univesity Press.

Jiménez de Aréchaga, Justino. (1906). La Libertad Politica: Fragmentos de un Curso de Derecho Constitucional. Montevideo: Editorial Tipográfica Nacional.

Lipset, Seymour Martin. (1959). "Some social requisites of democracy: economic development and political legitimacy”, American Political Science Review, 53, pp. 69-105.

Martínez, Martin C. (1964). Ante la Nueva Constitucion. Montevideo, Biblioteca Nacional.

Moore, Barrington (1966). Social Origins of Dictatorship and Democracy: Lord and Peasant in the Making 
of the Modern World. Boston, MA: Beacon Press.

Negretto Gabriel L. y Giancarlo Visconti (2017). "Electoral Reform Under Limited Party Competition: The Adoption of Proportional Representation in Latin America". Latin American Politics and Society, 60(1), pp. 27-51.

Pareja, Carlos (1988) "Asignaturas Pendientes en el Debate de la Reforma Política”, Cuadernos de Claeh, 47, pp. 19-46.

Pérez Antón, Romeo y Alfredo Castellanos (1980). El Pluralismo: Examen de la Experiencia Uruguaya 1830-1918. Montevideo: CLAEH.

Pérez Antón, R. (1988), "Cuatro Antagonismos sucesivos: la Concreta Instauración de la Democracia Uruguaya”, Revista Uruguaya de Ciencia Política, 2, pp. 41-59.

Pivel Devoto, Juan E. (1994). Historia de los Partidos Politicos en el Uruguay (2 tomos). Montevideo: Cámara de Representantes.

Ramírez, Juan A. (1976). Dos ensayos constitucionales. Montevideo: Ministerio de Instrucción Pública y Social.

Rokkan, Stein (1970). Citizens, Elections, and Parties: Approaches to the Comparative Study of the Process of Development. Oslo: Universitetsforlaget.

Schmitter, Philippe C. y Terry L. Karl (1993). "Qué es y qué no es la democracia”, Sistema, 116, pp. 17-30.

Wills-Otero, Laura (2009). "Electoral Systems in Latin America: Explaining the Adoption of Proportional Representation Systems During the Twentieth Century", Latin American Politics and Society, 51(3), pp. 33-58. 